\title{
Cyamella Sesamoiditis: A Rare Cause of Posterolateral Corner Knee Pain
}

\author{
Niharika Prasad ${ }^{1}$, Manchikanti Venkatesh ${ }^{2}$, Kopuri Ajay Abishek ${ }^{3}$ \\ ${ }^{1}$ Assistant Professor, Department of Radiology, Dr. D.Y. Patil Medical College, Hospital \& Research Center, Pune, Maharashtra, \\ ${ }^{2}$ Assistant Professor, Department of Radiology, Narayana Medical College, Nellore, Andhra Pradesh, ${ }^{3}$ Junior Resident, \\ Department of Radiology, Katuri Medical College, Guntur, Andhra Pradesh, India
}

Corresponding author: Dr. Manchikanti Venkatesh, Department of Radiology, Narayana Medical College, Nellore, Andhra Pradesh, India

DOI: http://dx.doi.org/10.21276/ijcmsr.2020.5.2.26

How to cite this article: Niharika Prasad, Manchikanti Venkatesh, Kopuri Ajay Abishek. Cyamella sesamoiditis: a rare cause of posterolateral corner knee pain. International Journal of Contemporary Medicine Surgery and Radiology. 2020;5(2):B106-B108.

\section{A B S T R A C T}

Introduction: Sesamoid bones causing pain is a rare phenomenon. Cyamella is a sesamoid bone located in popliteus tendon. Sesamoiditis of cyamella is a rare manifestation.

Case Report: We report a classic case of sesamoiditis of cyamella in a 50 year old male with classic features diagnosed on MRI with illustrations.

Conclusion: Sesamoiditis of cyamella is a rare cause of posterolateral corner knee pain which may remain undiagnosed if not properly evaluated with MRI. MRI plays a major role in identifying this rare cause of posterolateral knee pain.

Keywords: Cyamella, Popliteal Tendon, Sesamoiditis, Posterolateral Corner Knee Pain, MRI Knee

\section{INTRODUCTION}

Accessory ossicles are inconstant, well-formed bones, not arising from fractures or other pathological process. They result from unfused ossification centers, which can form free ossicles, sesamoid bones or bipartite ossicles. It is important to recognize them as normal variant and not to mistake them for pathologies. A few common examples are Os Vesalianum Pedis (adjacent to base of fifth metatarsal), bipartite scaphoid, Os Trigonum (posterior to talus), Os Acromiale (distal to acromion process), Os Peroneum (in Peroneus longus tendon), accessory navicular bone and bipartite patella. ${ }^{1}$

Sometimes, these may be symptomatic, due to trauma, infection, inflammation and degeneration. The various modalities useful in diagnosis include radiographs, ultrasonography, scintigraphy, computed tomography (CT) and magnetic resonance imaging (MRI). ${ }^{2}$ Sesamoid bones are partially or fully embedded in a corresponding tendon and develop from their own ossification center. ${ }^{3}$ Cyamella, described in this case report, is a rare sesamoid bone of the popliteus tendon.

\section{CASE REPORT}

A fifty-year-old male presented with pain in the posterolateral compartment of right knee since two weeks. He had no similar complaint in the other joints. He could not recall a previous fall. There was no history of surgery in the past. On examination, there was no redness or swelling over the right knee. However, there was localized tenderness over the medial and posterolateral aspect of the knee.
A plain radiograph was taken in another institution, which revealed a rounded, well-defined osseous body adjacent to the lateral tibial condyle. CT scan of the right knee was also performed, which confirmed it to be a sesamoid bone. No fracture was seen in the bones. Right minimal knee joint effusion was present. These images were not available with the patient. He was subjected to a plain MRI of knee in our institution on a 1.5 Tesla scanner. Routine MRI knee protocol was performed including axial, coronal and sagittal proton density, fat suppressed sequences and coronal T1\& axial T2 weighted sequences. They revealed a small wellcorticated, osseous body measuring $7 \times 6 \mathrm{~mm}$ in the popliteus tendon (Figure 1). This incidental finding was consistent with Cyamella. Fat suppressed proton density images

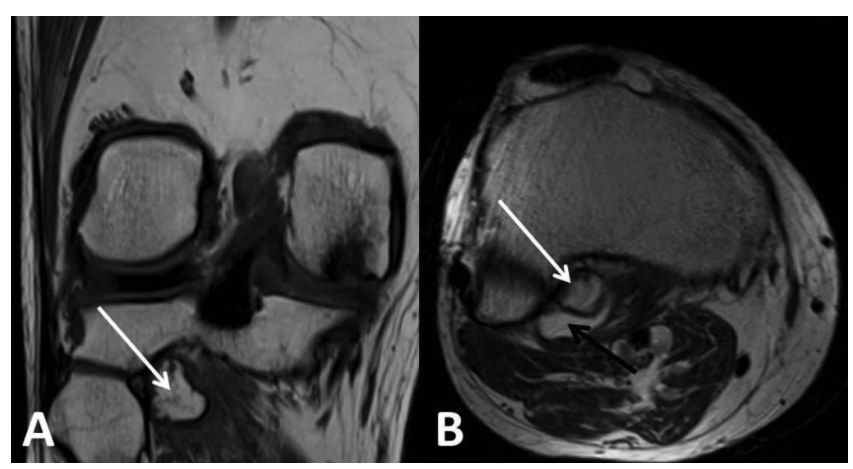

Figure-1: Coronal T1 weighted image (A) showing a well corticated osseous body (white arrow) in the popliteus tendon, Axial T2 weighted image (B) showing the osseous body (white arrow) with surrounding fluid (black arrow) 


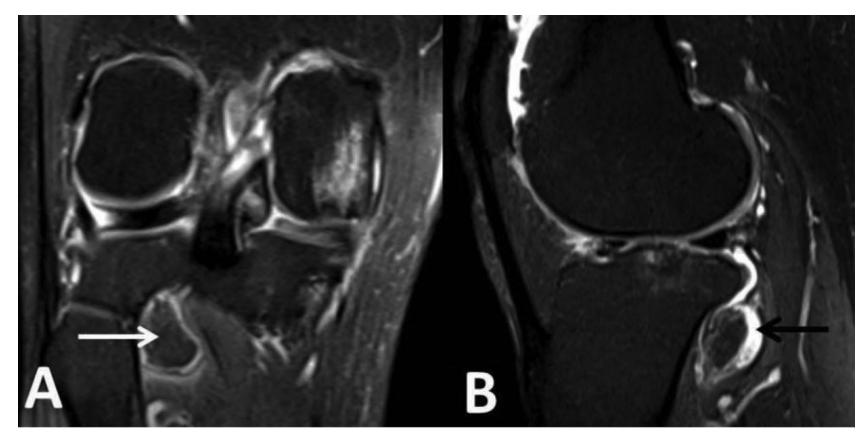

Figure-2: Coronal PDFS images (A) showing osseous body (white arrow) in the popliteal tendon with surrounding fluid; Sagittal PDFS image (B) showing peri-osseous fluid rim (black arrow) with marrow edema within the osseous bodysuggestive of Cyamella with features of sesamoiditis.

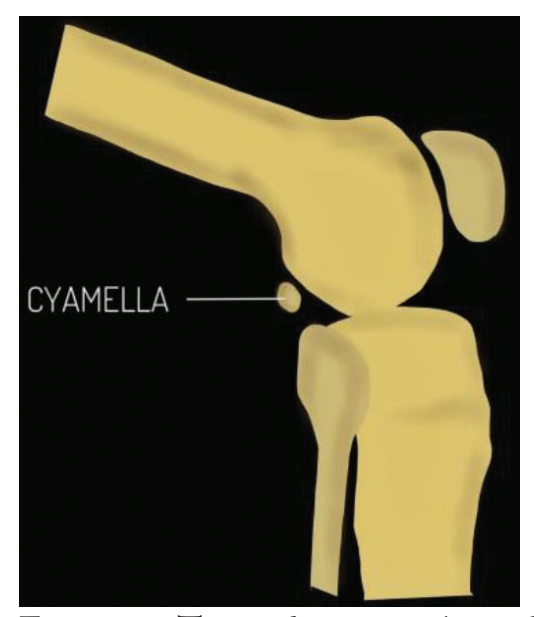

Figure-3: Three dimensional model showing sesamoid Cyamella in the posterolateral aspect of the knee. Source: Original

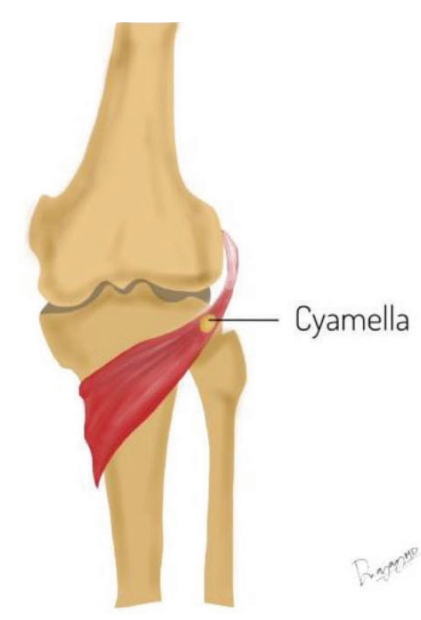

Figure-4: Illustrative image showing Cyamella embedded within the popliteus tendon. Source: Original

(Figure 2) demonstrated hyperintensity suggestive of bone marrow edema in the Cyamella with rim of surrounding fluid extending along the popliteus tendon. There was edema involving medial condyle of femur and medial tibial plateaulikely stress related. Mild suprapatellar joint effusion was present.

The patient was managed conservatively. Advice on rest and avoidance of excess weight bearing was given. The patient improved and recovered fully without the need of surgery.

\section{DISCUSSION}

Sesamoids and accessory ossicles are usually small, wellcorticated bodies located close to a bone or joint and may be bipartite or multipartite. They are found incidentally, however occasionally they may be symptomatic due to fracture or inflammation. The function of Sesamoids is to protect tendon from injury by reduction of friction., ${ }^{4,5}$

These are common in the hand and feet. Cyamella is a sesamoid which is found in the popliteus tendon of the knee (Figure 3 and 4). Differential diagnosis can include- fracture fragment, loose body, detached osteophyte and heterotopic ossification. ${ }^{6}$ History of trauma should be enquired for. Degenerative changes and other similar osseous bodies should be looked for on imaging. Sesamoid will usually be an incidental, asymptomatic finding. In above case, the classical location of Cyamella was useful in diagnosis. Peri articular calcification is outside the joint. Absence of soft tissue on radiograph and cross sectional imaging ruled out neoplasm. There was no bony defect seen in adjacent tibia and no cortical irregularity. Conservative treatment has been proved effective in few cases of sesamoiditis.,

\section{CONCLUSION}

Sesamoiditis should be included in the list of differential diagnosis for knee snapping and chronic knee pain with instability and locking. However, the presentation may be non-specific in many cases and there is no consensus in literature regarding specific management. ${ }^{7,8,9,10}$

\section{REFERENCES}

1. Vaz A, Trippia CR. Small but troublesome: accessory ossicles with clinical significance. Radiologia brasileira. 2018;51(4):248-56.

2. Nwawka OK, Hayashi D, Diaz LE, Goud AR, Arndt WF, Roemer FW, Malguria N, Guermazi A. Sesamoids and accessory ossicles of the foot: anatomical variability and related pathology. Insights into imaging. 2013;4(5):581-93.

3. Coskun N, Yuksel M, Cevener M, Arican RY, Ozdemir H, Bircan O, Sindel T, Ilgi S, Sindel M. Incidence of accessory ossicles and sesamoid bones in the feet: a radiographic study of the Turkish subjects. Surgical and radiologic anatomy. 2009;31(1):19.

4. Nwawka OK, Hayashi D, Diaz LE, Goud AR, Arndt WF, Roemer FW, Malguria N, Guermazi A. Sesamoids and accessory ossicles of the foot: anatomical variability and related pathology. Insights into imaging. 2013;4(5):581-93.

5. Chagas-Neto FA, Souza BN, Nogueira-Barbosa $\mathrm{MH}$. Painful os peroneum syndrome: underdiagnosed condition in the lateral midfoot pain. Case reports in radiology. 2016;2016.

6. Khanna V, Maldjian C. The cyamella, a lost sesamoid: normal variant or posterolateral corner anomaly? Radiology case reports. 2014;9(1):907.

7. Benthien JP, Brunner A. A symptomatic sesamoid bone in the popliteus muscle (cyamella). Musculoskeletal 
surgery. 2010;94(3):141-4.

8. Su S, Lu Y, Chen Y, Li Z. A symptomatic cyamella in the popliteus tendon causing snapping knee: a case report and literature review. BMC musculoskeletal disorders. 2019;20(1):495.

9. Aparisi MG, Aparisi F, Bartoloni A, Ferrando MF, Battista G, Guglielmi G, Bazzocchi A. Anatomical variation in the ankle and foot: from incidental finding to inductor of pathology. Part I: ankle and hindfoot. Insights into imaging. 2019;10(1):74-74.

10. Vora BM, Wong BS. Common accessory ossicles of the foot: imaging features, pitfalls and associated pathology. Singapore medical journal. 2018;59(4):183.

Source of Support: Nil; Conflict of Interest: None

Submitted: 15-04-2020; Accepted: 17-05-2020; Published online: 30-06-2020 